\section{CRYSTALLOGRAPHIC COMMUNICATIONS}

ISSN 2056-9890

\section{Crystal structure of $4,4^{\prime}, 4^{\prime \prime}$-(1,3,5-tria- zine-2,4,6-triyl)tripyridinium trichloride 2.5-hydrate}

Bo-Kai Ling, ${ }^{a}$ Xiao-Long Feng, ${ }^{b}$ Yang Lib* and Tian-Gang Luan $^{\mathrm{a}}$

${ }^{a}$ School of Marine Science, Sun Yat-Sen University, Guangzhou 510275, People's Republic of China, and ${ }^{\mathbf{b}}$ Instrumental Analysis and Research Center, Sun Yat-Sen University, Guangzhou 510275, People's Republic of China. *Correspondence e-mail: liyang223@mail.sysu.edu.cn

Received 18 September 2015; accepted 28 September 2015

Edited by D.-J. Xu, Zhejiang University (Yuquan Campus), China

The asymmetric unit of the title compound, $\mathrm{C}_{18} \mathrm{H}_{15} \mathrm{~N}_{6}{ }^{3+}$.$3 \mathrm{Cl}^{-} \cdot 2.5 \mathrm{H}_{2} \mathrm{O}$, contains two independent (1,3,5-triazine-2,4,6triyl)tripyridinium cations. Both cations are approximately planar, the r.m.s. deviations of fitted non-H atoms being 0.045 and $0.051 \AA$. In the crystal, extensive $\mathrm{O}-\mathrm{H} \cdots \mathrm{Cl}, \mathrm{O}-\mathrm{H} \cdots \mathrm{O}$, $\mathrm{N}-\mathrm{H} \cdots \mathrm{Cl}$ and $\mathrm{N}-\mathrm{H} \cdots \mathrm{O}$ hydrogen bonds and weak $\mathrm{C}-$ $\mathrm{H} \cdots \mathrm{Cl}$ and $\mathrm{C}-\mathrm{H} \cdots \mathrm{O}$ interactions link the organic cations, $\mathrm{Cl}^{-}$anions and water molecules into a three-dimensional supramolecular architecture. $\pi-\pi$ stacking between the pyridine rings of adjacent cations is also observed, the centroid-to-centroid distance being 3.7578 (8) A.

Keywords: crystal structure; 1,3,5-triazine; trichloride; hydrogen bonding; $\pi-\pi$ interactions.

CCDC reference: 1427933

\section{Related literature}

For applications of 2,4,6-tris(pyridin-4-yl)-1,3,5-triazine, see: Yoshizawa et al. (2006); Inokuma et al. (2011, 2013). For the crystal structure of 2,4,6-tris(pyridin-4-yl)-1,3,5-triazine (TPT), see: Janczak et al. (2003). For the crystal structure of (1,3,5-triazine-2,4,6-triyl)tripyridinium nitrate, see: Zhu et al. (2007).<smiles></smiles>

\section{Experimental}

\subsection{Crystal data}

$2 \mathrm{C}_{18} \mathrm{H}_{15} \mathrm{~N}_{6}{ }^{3+} \cdot 6 \mathrm{Cl}^{-} \cdot 5 \mathrm{H}_{2} \mathrm{O}$

$M_{r}=933.50$

Monoclinic $P 2_{1} / c$

$a=10.6042(1) \AA$

$b=14.6447$ (1) $\AA$

$c=27.7906(3) \AA$

$\beta=98.310(1)^{\circ}$

\subsection{Data collection}

Agilent Xcalibur Atlas Gemini ultra diffractometer

Absorption correction: multi-scan (CrysAlis PRO; Agilent, 2014)

$T_{\min }=0.575, T_{\max }=1.000$

\subsection{Refinement}

$R\left[F^{2}>2 \sigma\left(F^{2}\right)\right]=0.026$

$w R\left(F^{2}\right)=0.076$

$S=1.05$

7278 reflections

572 parameters
$V=4270.44(7) \AA^{3}$

$Z=4$

$\mathrm{Cu} K \alpha$ radiation

$\mu=4.15 \mathrm{~mm}^{-1}$

$T=150 \mathrm{~K}$

$0.50 \times 0.20 \times 0.10 \mathrm{~mm}$
27170 measured reflections 7278 independent reflections 6654 reflections with $I>2 \sigma(I)$ $R_{\text {int }}=0.020$

Table 1

Hydrogen-bond geometry $\left(\AA{ }^{\circ}\right)$.

\begin{tabular}{|c|c|c|c|c|}
\hline$D-\mathrm{H} \cdots A$ & $D-\mathrm{H}$ & $\mathrm{H} \cdots A$ & $D \cdots A$ & $D-\mathrm{H} \cdots A$ \\
\hline $\mathrm{O} 1-\mathrm{H} 1 A \cdots \mathrm{Cl}_{0} 3^{\mathrm{i}}$ & $0.77(2)$ & $2.47(2)$ & $3.2302(15)$ & $171(2)$ \\
\hline $\mathrm{O} 1-\mathrm{H} 1 B \cdots \mathrm{Cl} 04$ & $0.87(2)$ & $2.32(2)$ & $3.1856(14)$ & $175(2)$ \\
\hline $\mathrm{O} 2-\mathrm{H} 2 A \cdots \mathrm{Cl} 04$ & $0.86(2)$ & $2.28(2)$ & $3.1124(15)$ & $163.2(18)$ \\
\hline $\mathrm{O} 2-\mathrm{H} 2 B \cdots \mathrm{Cl} 05$ & $0.84(2)$ & $2.22(2)$ & $3.0515(13)$ & $170(2)$ \\
\hline $\mathrm{O} 3-\mathrm{H} 3 A \cdots \mathrm{Cl}^{2} 6^{\mathrm{ii}}$ & $0.84(2)$ & $2.22(2)$ & $3.0379(13)$ & $164.5(18)$ \\
\hline $\mathrm{O} 3-\mathrm{H} 3 B \cdots \mathrm{O} 2$ & $0.84(2)$ & $1.91(2)$ & $2.7426(17)$ & $176(2)$ \\
\hline $\mathrm{O} 4-\mathrm{H} 4 A \cdots \mathrm{Cl} 05^{\mathrm{iii}}$ & $0.78(2)$ & $2.36(2)$ & $3.1356(15)$ & $172(2)$ \\
\hline $\mathrm{O} 4-\mathrm{H} 4 B \cdots \mathrm{Cl} 03$ & $0.83(3)$ & $2.47(3)$ & $3.2626(14)$ & $162(3)$ \\
\hline $\mathrm{O} 5-\mathrm{H} 5 A \cdots \mathrm{Cl} 06$ & $0.92(2)$ & $2.12(2)$ & $2.9973(12)$ & $157.4(17)$ \\
\hline $\mathrm{O} 5-\mathrm{H} 5 B \cdots \mathrm{Cl} 03$ & $0.81(2)$ & $2.24(2)$ & $3.0466(12)$ & $173(2)$ \\
\hline $\mathrm{N} 1-\mathrm{H} 01 \cdots \mathrm{Cl} 01^{\text {iv }}$ & 0.86 & 2.24 & $3.0678(12)$ & 161 \\
\hline $\mathrm{N} 2-\mathrm{H} 02 \cdots \mathrm{O} 5$ & 0.86 & 1.77 & $2.5985(16)$ & 162 \\
\hline $\mathrm{N} 3-\mathrm{H} 03 \cdots \mathrm{Cl} 01$ & 0.86 & 2.23 & $3.0405(12)$ & 158 \\
\hline N7-H07..O3 & 0.86 & 1.84 & $2.6472(16)$ & 155 \\
\hline $\mathrm{N} 8-\mathrm{H} 08 \cdots \mathrm{Cl} 02^{\text {iv }}$ & 0.86 & 2.25 & $3.0732(12)$ & 159 \\
\hline $\mathrm{N} 9-\mathrm{H} 09 \ldots \mathrm{Cl} 02$ & 0.86 & 2.19 & $3.0337(12)$ & 166 \\
\hline $\mathrm{C} 1-\mathrm{H} 1 \cdots \mathrm{Cl} 03^{\mathrm{v}}$ & 0.93 & 2.57 & $3.4995(15)$ & 174 \\
\hline $\mathrm{C} 4-\mathrm{H} 4 \ldots \mathrm{Cl} 04$ & 0.93 & 2.61 & $3.4871(14)$ & 157 \\
\hline $\mathrm{C} 5-\mathrm{H} 5 \cdots \mathrm{O} 1$ & 0.93 & 2.38 & $3.2614(19)$ & 158 \\
\hline $\mathrm{C} 9-\mathrm{H} 9 \cdots \mathrm{Cl}^{2} 6^{\mathrm{v}}$ & 0.93 & 2.70 & $3.4071(15)$ & 134 \\
\hline
\end{tabular}

$\mathrm{H}$ atoms treated by a mixture of independent and constrained refinement

$\Delta \rho_{\max }=0.26$ e $\AA^{-3}$

$\Delta \rho_{\min }=-0.25 \mathrm{e}^{-3}$ 


\begin{tabular}{|c|c|c|c|c|}
\hline$D-\mathrm{H} \cdots A$ & $D-\mathrm{H}$ & $\mathrm{H} \cdots A$ & $D \cdots A$ & $D-\mathrm{H} \cdots A$ \\
\hline$\overline{\mathrm{C}} 10-\mathrm{H} 10 \cdots \mathrm{O} 4$ & 0.93 & 2.54 & $3.370(2)$ & 148 \\
\hline $\mathrm{C} 11-\mathrm{H} 11 \cdots \mathrm{Cl} 02^{\mathrm{vi}}$ & 0.93 & 2.61 & $3.5114(15)$ & 163 \\
\hline $\mathrm{C} 12-\mathrm{H} 12 \cdots \mathrm{Cl} 04$ & 0.93 & 2.64 & $3.4992(15)$ & 154 \\
\hline $\mathrm{C} 15-\mathrm{H} 15 \cdots \mathrm{Cl} 3^{\mathrm{vii}}$ & 0.93 & 2.54 & $3.3157(16)$ & 141 \\
\hline $\mathrm{C} 21-\mathrm{H} 21 \cdots \mathrm{Cl} 05$ & 0.93 & 2.74 & $3.5424(15)$ & 146 \\
\hline $\mathrm{C} 22-\mathrm{H} 22 \cdots \mathrm{Cl} 06^{\mathrm{v}}$ & 0.93 & 2.72 & $3.3883(15)$ & 130 \\
\hline $\mathrm{C} 24-\mathrm{H} 24 \cdots \mathrm{Cl} 05^{\mathrm{viii}}$ & 0.93 & 2.63 & $3.4519(15)$ & 147 \\
\hline $\mathrm{C} 26-\mathrm{H} 26 \cdots \mathrm{Cl} 04^{\mathrm{ix}}$ & 0.93 & 2.53 & $3.3669(15)$ & 149 \\
\hline $\mathrm{C} 30-\mathrm{H} 30 \cdots \mathrm{O} 2^{\mathrm{x}}$ & 0.93 & 2.31 & $3.2331(19)$ & 174 \\
\hline $\mathrm{C} 31-\mathrm{H} 31 \cdots \mathrm{O} 2^{\text {viii }}$ & 0.93 & 2.50 & $3.3320(19)$ & 149 \\
\hline $\mathrm{C} 34-\mathrm{H} 34 \cdots \mathrm{O} 1^{\mathrm{ix}}$ & 0.93 & 2.50 & $3.3706(19)$ & 156 \\
\hline $\mathrm{C} 35-\mathrm{H} 35 \cdots \mathrm{Cl} 01^{\mathrm{xi}}$ & 0.93 & 2.72 & $3.6254(14)$ & 166 \\
\hline
\end{tabular}

Symmetry codes: (i) $x+1,-y+\frac{1}{2}, z-\frac{1}{2}$; (ii) $x+1, y, z$; (iii) $x-1, y, z$; (iv) $x, y-1, z$; (v) $-x, y-\frac{1}{2},-z+\frac{3}{2}$; (vi) $\quad x+1,-y+\frac{3}{2}, z-\frac{1}{2}$; (vii) $-x, y+\frac{1}{2},-z+\frac{3}{2}$; (viii) $-x+1, y+\frac{1}{2},-z+\frac{3}{2} ; \quad$ (ix) $\quad x-1,-y+\frac{1}{2}, z+\frac{1}{2} ; \quad$ (x) $-x+1, y-\frac{1}{2},-z+\frac{3}{2} ; \quad$ (xi) $x-1,-y+\frac{3}{2}, z+\frac{1}{2}$.

Data collection: CrysAlis PRO (Agilent, 2014); cell refinement: CrysAlis PRO; data reduction: CrysAlis PRO; program(s) used to solve structure: SHELXS97 (Sheldrick, 2008); program(s) used to refine structure: SHELXL2014 (Sheldrick, 2015); molecular graphics: ORTEP-3 for Windows (Farrugia, 2012); software used to prepare material for publication: Win $G X$ (Farrugia, 2012).

\section{Acknowledgements}

This research was supported financially by the National Natural Science Foundation of China (NSFC grants No. 21277177 and 41473092), the Foundation for High-level Talents in Higher Education of Guangdong Province and the Administration of Ocean and Fisheries of Guangdong Province, China.

Supporting information for this paper is available from the IUCr electronic archives (Reference: XU5874).

\section{References}

Agilent (2014). CrysAlis PRO. Agilent Technologies, Yarnton, England. Farrugia, L. J. (2012). J. Appl. Cryst. 45, 849-854.

Inokuma, Y., Kawano, M. \& Fujita, M. (2011). Nat. Chem. 3, 349-358.

Inokuma, Y., Yoshioka, S., Ariyoshi, J., Arai, T., Hitora, Y., Takada, K., Matsunaga, S., Rissanen, K. \& Fujita, M. (2013). Nature, 495, 461-466. Janczak, J., Śledź, M. \& Kubiak, R. (2003). J. Mol. Struct. 659, 71-79.

Sheldrick, G. M. (2008). Acta Cryst. A64, 112-122.

Sheldrick, G. M. (2015). Acta Cryst. C71, 3-8.

Yoshizawa, M., Tamura, M. \& Fujita, M. (2006). Science, 312, 251-254.

Zhu, S.-R., Chen, W.-D., Zhao, H.-J., Shao, M. \& Li, M.-X. (2007). Acta Cryst. E63, o1344-o1346. 


\section{supporting information}

Acta Cryst. (2015). E71, o858-o859 [https://doi.org/10.1107/S2056989015018125]

Crystal structure of $4,4^{\prime}, 4^{\prime \prime}$-(1,3,5-triazine-2,4,6-triyl)tripyridinium trichloride 2.5-hydrate

\section{Bo-Kai Ling, Xiao-Long Feng, Yang Li and Tian-Gang Luan}

\section{S1. Comment}

2,4,6-Tris(4-pyridyl)-1,3,5-triazine (TPT), as a planar tridentate ligand for MOFs (metal-organic frameworks), has been designed for some useful crystals by reactions with metal ions. Due to its special triazine $\pi-\pi$ interaction, triangular plane geometry and tridentate $\mathrm{N}$ atoms coordinate, these crystals remarkable applications were discovered gradually such as molecular flask (Yoshizawa \& Fujita, 2006; Inokuma \& Fujita, 2011) and X-ray single-crystal diffraction carrier (Inokuma \& Fujita, 2013). The crystal structure of neutral tpt was reported by (Janczak \& Kubiak, 2003). The nitrate salt of TPT was published by Zhu (Zhu et al. 2007).

The crystal has a well layered form through $\pi$ - $\pi$ interaction and Hydrogen Bonds which is analogous to pure TPT crystal (Janczak \& Kubiak, 2003). In the crystal, every pyridine has protonized. $\mathrm{H}_{3} \mathrm{TPT}, \mathrm{Cl}^{-}$and $\mathrm{H}_{2} \mathrm{O}$ pack in a layer through ionic bonding and hydrogen-bonding.

\section{S2. Synthesis and crystallization}

Excess hydrochloric acid $(2 \mathrm{~mL})$ was added in pure TPT $(93 \mathrm{mg}, 0.3 \mathrm{mmol})$ in a $20 \mathrm{~mL}$ scintillation vial. With the dropwise addition of hydrochloric acid, solution was clear gradually. Then the mixture was put in an oven at $393 \mathrm{~K}$ for 10h. The colourless crystal will be found.

\section{S3. Refinement}

All $\mathrm{H}$ atoms for $\mathrm{C}$ and $\mathrm{N}$ atoms were geometrically fixed and allowed to ride on their parent $\mathrm{C}$ and $\mathrm{N}$ atoms, with $\mathrm{C}-\mathrm{H}=$ $0.93 \AA, \mathrm{N}-\mathrm{H}=0.86 \AA$ ) and with $U_{\text {iso }}(\mathrm{H})=1.2 U_{\text {eq }}(\mathrm{C}), U_{\text {iso }}(\mathrm{H})=1.2 U_{\text {eq }}(\mathrm{N})$. $\mathrm{H}$ atoms belonging to $\mathrm{H}_{2} \mathrm{O}$ groups were located in difference Fourier maps and refined isotropically. 
¿LO2

$e^{c l 01}$

6

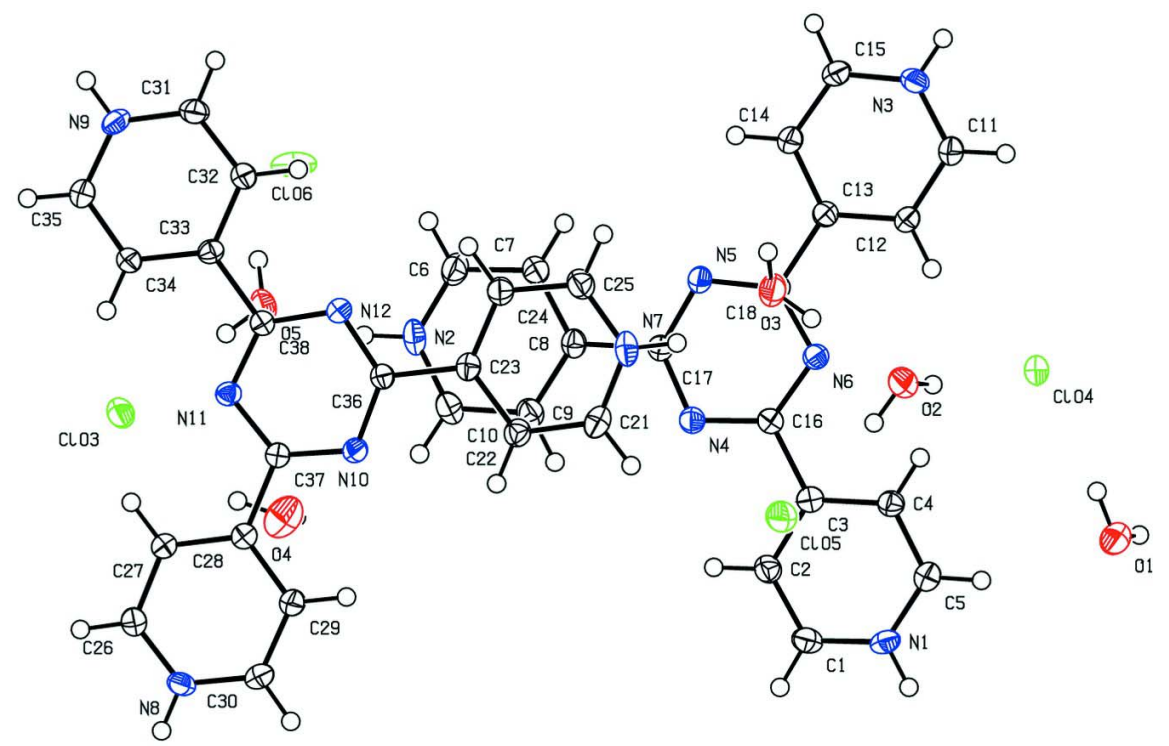

Figure 1

The molecular structure of the title compound, showing the atom labeling. Displacement ellipsoids are drawn at the $50 \%$ probability level.

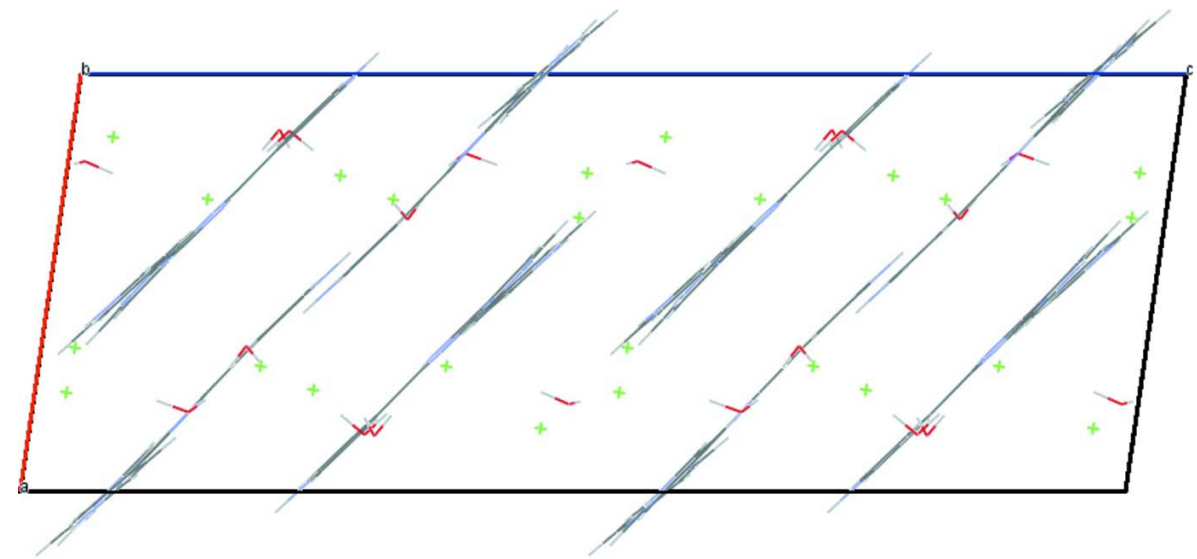

Figure 2

The crystal packing of the title compound viewed along the $b$ axis. Colour key: red indicates oxygen and green chlorine.

4,4',4"'-(1,3,5-Triazine-2,4,6-triyl)tripyridinium trichloride 2.5 -hydrate

Crystal data

$2 \mathrm{C}_{18} \mathrm{H}_{15} \mathrm{~N}_{6}{ }^{3+} \cdot 6 \mathrm{Cl}^{-} \cdot 5 \mathrm{H}_{2} \mathrm{O}$

$M_{r}=933.50$

Monoclinic, $P 2_{1} / c$

$a=10.6042(1) \AA$

$b=14.6447$ (1) $\AA$

$c=27.7906$ (3) $\AA$

$V=4270.44(7) \AA^{3}$

$Z=4$

$F(000)=1928$

$D_{\mathrm{x}}=1.452 \mathrm{Mg} \mathrm{m}^{-3}$

$\beta=98.310(1)^{\circ}$

$\mathrm{Cu} K \alpha$ radiation, $\lambda=1.54184 \AA$

Cell parameters from 15012 reflections

$\theta=3.0-65.5^{\circ}$ 
$\mu=4.15 \mathrm{~mm}^{-1}$

$T=150 \mathrm{~K}$

\section{Data collection}

Agilent Xcalibur Atlas Gemini ultra diffractometer

Detector resolution: 10.5058 pixels $\mathrm{mm}^{-1}$

$\omega$ scans

Absorption correction: multi-scan

(CrysAlis PRO; Agilent, 2014)

$T_{\min }=0.575, T_{\max }=1.000$

27170 measured reflections

\section{Refinement}

Refinement on $F^{2}$

Least-squares matrix: full

$R\left[F^{2}>2 \sigma\left(F^{2}\right)\right]=0.026$

$w R\left(F^{2}\right)=0.076$

$S=1.05$

7278 reflections

572 parameters

0 restraints
Rod, colourless

$0.50 \times 0.20 \times 0.10 \mathrm{~mm}$

7278 independent reflections

6654 reflections with $I>2 \sigma(I)$

$R_{\text {int }}=0.020$

$\theta_{\text {max }}=65.6^{\circ}, \theta_{\min }=3.2^{\circ}$

$h=-12 \rightarrow 12$

$k=-11 \rightarrow 16$

$l=-32 \rightarrow 31$

Hydrogen site location: inferred from neighbouring sites

$\mathrm{H}$ atoms treated by a mixture of independent and constrained refinement

$w=1 /\left[\sigma^{2}\left(F_{\mathrm{o}}{ }^{2}\right)+(0.0455 P)^{2}+1.0459 P\right]$ where $P=\left(F_{\mathrm{o}}^{2}+2 F_{\mathrm{c}}{ }^{2}\right) / 3$

$(\Delta / \sigma)_{\max }=0.002$

$\Delta \rho_{\max }=0.26 \mathrm{e} \AA^{-3}$

$\Delta \rho_{\min }=-0.25$ e $\AA^{-3}$

Special details

Geometry. All e.s.d.'s (except the e.s.d. in the dihedral angle between two 1.s. planes) are estimated using the full covariance matrix. The cell e.s.d.'s are taken into account individually in the estimation of e.s.d.'s in distances, angles and torsion angles; correlations between e.s.d.'s in cell parameters are only used when they are defined by crystal symmetry. An approximate (isotropic) treatment of cell e.s.d.'s is used for estimating e.s.d.'s involving l.s. planes.

Fractional atomic coordinates and isotropic or equivalent isotropic displacement parameters $\left(\AA^{2}\right)$

\begin{tabular}{lllll}
\hline & $x$ & $y$ & $z$ & $U_{\text {iso }} * / U_{\text {eq }}$ \\
\hline C101 & $0.65529(3)$ & $0.87819(2)$ & $0.53046(2)$ & $0.02080(9)$ \\
C102 & $-0.15165(3)$ & $0.86725(2)$ & $0.96259(2)$ & $0.02038(9)$ \\
C103 & $-0.30029(3)$ & $0.33680(2)$ & $0.86906(2)$ & $0.02704(9)$ \\
C104 & $0.76231(3)$ & $0.34758(2)$ & $0.52883(2)$ & $0.02860(10)$ \\
C105 & $0.69917(4)$ & $0.19615(3)$ & $0.70100(2)$ & $0.03228(10)$ \\
C106 & $-0.24393(4)$ & $0.61127(3)$ & $0.75183(2)$ & $0.03989(12)$ \\
O5 & $-0.13385(10)$ & $0.45402(8)$ & $0.81302(4)$ & $0.0293(2)$ \\
O3 & $0.65114(11)$ & $0.45593(9)$ & $0.68539(4)$ & $0.0292(2)$ \\
O2 & $0.80923(11)$ & $0.34430(8)$ & $0.64199(5)$ & $0.0308(2)$ \\
O1 & $0.79029(12)$ & $0.14892(9)$ & $0.48489(5)$ & $0.0349(3)$ \\
N11 & $0.05285(10)$ & $0.36030(8)$ & $0.90020(4)$ & $0.0181(2)$ \\
N10 & $0.20086(10)$ & $0.29011(7)$ & $0.85541(4)$ & $0.0189(2)$ \\
N6 & $0.46303(11)$ & $0.37119(8)$ & $0.59643(4)$ & $0.0193(2)$ \\
N5 & $0.33219(11)$ & $0.46467(8)$ & $0.63822(4)$ & $0.0199(2)$ \\
O4 & $-0.13673(13)$ & $0.20960(9)$ & $0.80408(5)$ & $0.0434(3)$ \\
N4 & $0.32306(11)$ & $0.30340(8)$ & $0.64532(4)$ & $0.0204(2)$ \\
N12 & $0.18691(10)$ & $0.45145(8)$ & $0.85844(4)$ & $0.0190(2)$ \\
N9 & $-0.06099(11)$ & $0.67836(8)$ & $0.93866(4)$ & $0.0226(3)$ \\
& & & &
\end{tabular}




\begin{tabular}{|c|c|c|c|c|}
\hline H09 & -0.0926 & 0.7271 & 0.9494 & $0.027^{*}$ \\
\hline N3 & $0.57300(11)$ & $0.68432(8)$ & $0.54975(4)$ & $0.0229(3)$ \\
\hline H03 & 0.6040 & 0.7318 & 0.5375 & $0.027 *$ \\
\hline N1 & $0.52800(11)$ & $0.03964(8)$ & $0.57480(4)$ & $0.0224(3)$ \\
\hline H01 & 0.5519 & -0.0134 & 0.5663 & $0.027 *$ \\
\hline N8 & $-0.00911(11)$ & $0.02831(8)$ & $0.92431(4)$ & $0.0225(3)$ \\
\hline H08 & -0.0319 & -0.0247 & 0.9332 & $0.027 *$ \\
\hline N7 & $0.51720(11)$ & $0.39554(9)$ & $0.75219(4)$ & $0.0251(3)$ \\
\hline H07 & 0.5727 & 0.4001 & 0.7326 & $0.030^{*}$ \\
\hline N2 & $0.00613(11)$ & $0.41058(9)$ & $0.74708(4)$ & $0.0268(3)$ \\
\hline H02 & -0.0498 & 0.4151 & 0.7665 & $0.032 *$ \\
\hline $\mathrm{C} 16$ & $0.41129(12)$ & $0.29914(9)$ & $0.61556(5)$ & $0.0181(3)$ \\
\hline $\mathrm{C} 36$ & $0.23658(12)$ & $0.37382(9)$ & $0.84431(5)$ & $0.0173(3)$ \\
\hline C37 & $0.10782(12)$ & $0.28751(9)$ & $0.88325(5)$ & $0.0164(3)$ \\
\hline $\mathrm{C} 17$ & $0.28607(13)$ & $0.38778(9)$ & $0.65473(5)$ & $0.0186(3)$ \\
\hline $\mathrm{C} 38$ & $0.09613(12)$ & $0.44037(9)$ & $0.88672(5)$ & $0.0171(3)$ \\
\hline $\mathrm{C} 23$ & $0.33836(13)$ & $0.38155(9)$ & $0.81286(5)$ & $0.0191(3)$ \\
\hline $\mathrm{C} 18$ & $0.42064(12)$ & $0.45195(9)$ & $0.60929(5)$ & $0.0183(3)$ \\
\hline C33 & $0.04010(12)$ & $0.52483(9)$ & $0.90481(5)$ & $0.0181(3)$ \\
\hline $\mathrm{C} 28$ & $0.06487(12)$ & $0.19579(9)$ & $0.89691(5)$ & $0.0179(3)$ \\
\hline C34 & $-0.04614(13)$ & $0.51835(9)$ & $0.93800(5)$ & $0.0206(3)$ \\
\hline H34 & -0.0701 & 0.4616 & 0.9486 & $0.025^{*}$ \\
\hline $\mathrm{C} 8$ & $0.18526(13)$ & $0.39663(10)$ & $0.68655(5)$ & $0.0203(3)$ \\
\hline $\mathrm{C} 3$ & $0.45303(12)$ & $0.20713(9)$ & $0.60187(5)$ & $0.0190(3)$ \\
\hline $\mathrm{C} 12$ & $0.56434(13)$ & $0.52499(10)$ & $0.55698(5)$ & $0.0211(3)$ \\
\hline H12 & 0.5915 & 0.4673 & 0.5490 & $0.025^{*}$ \\
\hline $\mathrm{C} 22$ & $0.39093(13)$ & $0.30344(10)$ & $0.79539(5)$ & $0.0221(3)$ \\
\hline $\mathrm{H} 22$ & 0.3655 & 0.2459 & 0.8043 & $0.027 *$ \\
\hline $\mathrm{C} 4$ & $0.54831(13)$ & $0.19906(9)$ & $0.57262(5)$ & $0.0204(3)$ \\
\hline H4 & 0.5869 & 0.2509 & 0.5620 & $0.025^{*}$ \\
\hline $\mathrm{C} 27$ & $-0.02943(13)$ & $0.18762(9)$ & $0.92639(5)$ & $0.0214(3)$ \\
\hline $\mathrm{H} 27$ & -0.0680 & 0.2394 & 0.9371 & $0.026^{*}$ \\
\hline $\mathrm{C} 13$ & $0.47470(12)$ & $0.53481(9)$ & $0.58865(5)$ & $0.0189(3)$ \\
\hline $\mathrm{C} 2$ & $0.39638(14)$ & $0.12860(10)$ & $0.61731(5)$ & 0.0232 \\
\hline $\mathrm{H} 2$ & 0.3332 & 0.1329 & 0.6372 & $0.028 *$ \\
\hline C9 & $0.13433(13)$ & $0.31852(10)$ & $0.70484(5)$ & 0.0236 \\
\hline H9 & 0.1610 & 0.2609 & 0.6965 & $0.028^{*}$ \\
\hline $\mathrm{C} 7$ & $0.14280(14)$ & $0.48231(10)$ & $0.69901(5)$ & 0.0250 \\
\hline H7 & 0.1751 & 0.5352 & 0.6868 & $0.030 *$ \\
\hline $\mathrm{C} 31$ & $0.02052(14)$ & $0.68671(10)$ & $0.90647(5)$ & 0.0244 \\
\hline H31 & 0.0416 & 0.7444 & 0.8961 & $0.029^{*}$ \\
\hline $\mathrm{C} 5$ & $0.58482(13)$ & $0.11328(10)$ & $0.55959(5)$ & $0.0227(3)$ \\
\hline H5 & 0.6489 & 0.1068 & 0.5402 & $0.027 *$ \\
\hline C11 & $0.61224(13)$ & $0.60190(10)$ & $0.53762(5)$ & 0.0230 \\
\hline H11 & 0.6716 & 0.5965 & 0.5162 & $0.028 *$ \\
\hline $\mathrm{C} 24$ & $0.37914(13)$ & $0.46732(10)$ & $0.79972(5)$ & 0.0232 \\
\hline $\mathrm{H} 24$ & 0.3456 & 0.5202 & 0.8114 & $0.028 *$ \\
\hline $\mathrm{C} 29$ & $0.11984(14)$ & $0.11722(9)$ & $0.88081(5)$ & 0.0233 \\
\hline
\end{tabular}




$\begin{array}{lllll}\text { H29 } & 0.1817 & 0.1215 & 0.8603 & 0.028^{*} \\ \text { C1 } & 0.43502(14) & 0.04472(10) & 0.60282(5) & 0.0250(3) \\ \text { H1 } & 0.3971 & -0.0083 & 0.6124 & 0.030^{*} \\ \text { C26 } & -0.06542(14) & 0.10175(10) & 0.93967(5) & 0.0240(3) \\ \text { H26 } & -0.1288 & 0.0952 & 0.9593 & 0.029^{*} \\ \text { C30 } & 0.08193(14) & 0.03369(10) & 0.89543(5) & 0.0265(3) \\ \text { H30 } & 0.1190 & -0.0192 & 0.8854 & 0.032^{*} \\ \text { C32 } & 0.07329(13) & 0.61014(9) & 0.88867(5) & 0.0224(3) \\ \text { H32 } & 0.1301 & 0.6154 & 0.8663 & 0.027^{*} \\ \text { C35 } & -0.09538(13) & 0.59728(10) & 0.95483(5) & 0.0231(3) \\ \text { H35 } & -0.1522 & 0.5943 & 0.9773 & 0.028^{*} \\ \text { C25 } & 0.47006(14) & 0.47203(10) & 0.76910(5) & 0.0261(3) \\ \text { H25 } & 0.4989 & 0.5286 & 0.7601 & 0.031^{*} \\ \text { C6 } & 0.05204(14) & 0.48706(11) & 0.72970(5) & 0.0288(3) \\ \text { H6 } & 0.0225 & 0.5436 & 0.7384 & 0.035^{*} \\ \text { C14 } & 0.43625(14) & 0.62155(10) & 0.60020(6) & 0.0252(3) \\ \text { H14 } & 0.3764 & 0.6292 & 0.6213 & 0.030^{*} \\ \text { C15 } & 0.48771(14) & 0.69618(10) & 0.58015(6) & 0.0267(3) \\ \text { H15 } & 0.4632 & 0.7548 & 0.5877 & 0.032^{*} \\ \text { C21 } & 0.48130(14) & 0.31252(10) & 0.76462(5) & 0.0256(3) \\ \text { H21 } & 0.5171 & 0.2609 & 0.7526 & 0.031^{*} \\ \text { C10 } & 0.04389(14) & 0.32775(11) & 0.73544(5) & 0.0269(3) \\ \text { H10 } & 0.0092 & 0.2762 & 0.7480 & 0.032^{*} \\ \text { H3A } & 0.6891(18) & 0.5011(15) & 0.6995(7) & 0.040(5)^{*} \\ \text { H2A } & 0.7816(19) & 0.3391(13) & 0.6115(8) & 0.042(6)^{*} \\ \text { H5A } & -0.1779(18) & 0.5060(14) & 0.8017(7) & 0.042(5)^{*} \\ \text { H5B } & -0.183(2) & 0.4236(15) & 0.8260(8) & 0.048(6)^{*} \\ \text { H3B } & 0.7020(19) & 0.4219(14) & 0.6736(7) & 0.041(5)^{*} \\ \text { H1A } & 0.761(2) & 0.1505(14) & 0.4580(8) & 0.044(6)^{*} \\ \text { H1B } & 0.784(2) & 0.2044(17) & 0.4950(8) & 0.054(6)^{*} \\ \text { H2B } & 0.780(2) & 0.2994(16) & 0.6554(8) & 0.058(7)^{*} \\ \text { H4A } & -0.184(2) & 0.2070(14) & 0.7798(8) & 0.047(6)^{*} \\ \text { H4B } & -0.187(3) & 0.2301(19) & 0.8215(10) & 0.081(9)^{*} \\ & & & & \end{array}$

Atomic displacement parameters $\left(\AA^{2}\right)$

\begin{tabular}{lllllll}
\hline & $U^{11}$ & $U^{22}$ & $U^{33}$ & $U^{12}$ & $U^{13}$ & $U^{23}$ \\
\hline C101 & $0.02249(17)$ & $0.01723(17)$ & $0.02277(17)$ & $-0.00011(12)$ & $0.00355(13)$ & $-0.00023(12)$ \\
C102 & $0.02001(16)$ & $0.01681(16)$ & $0.02501(17)$ & $0.00094(12)$ & $0.00562(13)$ & $0.00004(12)$ \\
C103 & $0.02599(18)$ & $0.02703(19)$ & $0.02979(19)$ & $-0.00023(14)$ & $0.00972(14)$ & $0.00424(14)$ \\
C104 & $0.03331(19)$ & $0.02561(19)$ & $0.03073(19)$ & $0.00059(14)$ & $0.01765(15)$ & $0.00042(14)$ \\
C105 & $0.0433(2)$ & $0.0277(2)$ & $0.02738(19)$ & $-0.00284(16)$ & $0.01019(16)$ & $-0.00100(14)$ \\
C106 & $0.0475(3)$ & $0.0177(2)$ & $0.0515(3)$ & $0.00120(15)$ & $-0.0030(2)$ & $0.00063(15)$ \\
O5 & $0.0277(6)$ & $0.0328(6)$ & $0.0302(6)$ & $0.0034(5)$ & $0.0133(5)$ & $0.0089(5)$ \\
O3 & $0.0305(6)$ & $0.0310(6)$ & $0.0284(6)$ & $-0.0026(5)$ & $0.0117(5)$ & $-0.0055(5)$ \\
O2 & $0.0363(6)$ & $0.0271(6)$ & $0.0311(6)$ & $-0.0062(5)$ & $0.0117(5)$ & $-0.0014(5)$ \\
O1 & $0.0458(7)$ & $0.0299(7)$ & $0.0313(7)$ & $0.0061(5)$ & $0.0130(6)$ & $-0.0008(5)$ \\
N11 & $0.0168(5)$ & $0.0166(6)$ & $0.0207(6)$ & $0.0013(4)$ & $0.0023(4)$ & $0.0007(4)$
\end{tabular}




\begin{tabular}{|c|c|c|c|c|c|c|}
\hline N10 & $0.0193(6)$ & $0.0178(6)$ & $0.0200(6)$ & $-0.0003(4)$ & $0.0041(4)$ & $-0.0017(4)$ \\
\hline N6 & $0.0177(5)$ & $0.0182(6)$ & $0.0223(6)$ & $-0.0009(4)$ & $0.0040(5)$ & $-0.0013(5)$ \\
\hline N5 & $0.0196(6)$ & 0.0209 (6) & $0.0200(6)$ & $0.0002(5)$ & $0.0058(4)$ & $-0.0012(5)$ \\
\hline $\mathrm{O} 4$ & $0.0417(7)$ & $0.0501(8)$ & $0.0365(7)$ & $0.0121(6)$ & $-0.0004(6)$ & $-0.0063(6)$ \\
\hline N4 & $0.0207(6)$ & $0.0205(6)$ & $0.0204(6)$ & $0.0020(5)$ & $0.0043(5)$ & $0.0019(5)$ \\
\hline N12 & $0.0197(6)$ & $0.0176(6)$ & $0.0202(6)$ & 0.0011 (4) & 0.0044 (4) & -0.0004 \\
\hline N9 & $0.0227(6)$ & $0.0178(6)$ & $0.0277(6)$ & $0.0037(5)$ & $0.0053(5)$ & $-0.0045(5)$ \\
\hline N3 & $0.0235(6)$ & 0.0177 (6) & $0.0280(6)$ & $-0.0024(5)$ & $0.0060(5)$ & $0.0029(5)$ \\
\hline N1 & $0.0266(6)$ & $0.0159(6)$ & $0.0241(6)$ & $0.0045(5)$ & $0.0018(5)$ & $-0.0009(5)$ \\
\hline N8 & $0.0291(6)$ & $0.0159(6)$ & $0.0228(6)$ & $-0.0031(5)$ & $0.0053(5)$ & $0.0019(5)$ \\
\hline N7 & $0.0201(6)$ & $0.0362(7)$ & $0.0204(6)$ & $-0.0038(5)$ & 0.0077 (5) & $-0.0024(5)$ \\
\hline N2 & $0.0217(6)$ & $0.0391(7)$ & $0.0214(6)$ & $0.0031(5)$ & 0.0095 (5) & $0.0048(5)$ \\
\hline $\mathrm{C} 16$ & $0.0168(6)$ & $0.0188(7)$ & $0.0182(6)$ & $-0.0002(5)$ & $0.0010(5)$ & $0.0011(5)$ \\
\hline $\mathrm{C} 36$ & $0.0170(6)$ & 0.0185 (7) & $0.0163(6)$ & $-0.0004(5)$ & $0.0021(5)$ & $0.0000(5)$ \\
\hline C37 & $0.0152(6)$ & $0.0180(7)$ & $0.0157(6)$ & $0.0001(5)$ & 0.0007 (5) & $-0.0001(5)$ \\
\hline $\mathrm{C} 17$ & $0.0183(6)$ & $0.0198(7)$ & $0.0176(6)$ & $0.0013(5)$ & $0.0021(5)$ & $0.0010(5)$ \\
\hline C38 & $0.0161(6)$ & $0.0181(7)$ & $0.0170(6)$ & $0.0000(5)$ & $0.0018(5)$ & $0.0007(5)$ \\
\hline $\mathrm{C} 23$ & $0.0172(6)$ & $0.0228(7)$ & $0.0171(6)$ & $-0.0013(5)$ & $0.0019(5)$ & $-0.0023(5)$ \\
\hline $\mathrm{C} 18$ & $0.0168(6)$ & $0.0185(7)$ & $0.0192(6)$ & $0.0002(5)$ & $0.0016(5)$ & $-0.0010(5)$ \\
\hline C33 & $0.0170(6)$ & $0.0169(7)$ & $0.0198(6)$ & $0.0002(5)$ & $0.0014(5)$ & $-0.0014(5)$ \\
\hline $\mathrm{C} 28$ & $0.0172(6)$ & $0.0193(7)$ & $0.0164(6)$ & $0.0010(5)$ & $-0.0002(5)$ & $-0.0002(5)$ \\
\hline C34 & $0.0208(7)$ & $0.0181(7)$ & $0.0238(7)$ & $-0.0007(5)$ & $0.0070(5)$ & $0.0013(5)$ \\
\hline C8 & $0.0186(7)$ & $0.0252(7)$ & $0.0171(6)$ & $0.0023(6)$ & $0.0025(5)$ & $0.0015(5)$ \\
\hline $\mathrm{C} 3$ & $0.0175(6)$ & $0.0195(7)$ & $0.0191(6)$ & $0.0004(5)$ & $-0.0002(5)$ & $-0.0001(5)$ \\
\hline $\mathrm{C} 12$ & $0.0216(7)$ & $0.0188(7)$ & $0.0236(7)$ & $0.0015(5)$ & $0.0064(6)$ & $-0.0017(5)$ \\
\hline $\mathrm{C} 22$ & $0.0217(7)$ & $0.0228(7)$ & $0.0222(7)$ & $-0.0015(6)$ & $0.0042(6)$ & $-0.0025(6)$ \\
\hline $\mathrm{C} 4$ & $0.0205(7)$ & $0.0196(7)$ & $0.0212(7)$ & $-0.0009(5)$ & $0.0031(5)$ & $-0.0008(5)$ \\
\hline $\mathrm{C} 27$ & $0.0247(7)$ & $0.0181(7)$ & $0.0224(7)$ & $0.0019(6)$ & $0.0071(6)$ & $-0.0002(5)$ \\
\hline $\mathrm{C} 13$ & $0.0178(6)$ & $0.0190(7)$ & $0.0198(7)$ & $-0.0007(5)$ & $0.0025(5)$ & $-0.0007(5)$ \\
\hline $\mathrm{C} 2$ & $0.0216(7)$ & $0.0228(7)$ & $0.0260(7)$ & $0.0005(6)$ & $0.0059(6)$ & $0.0025(6)$ \\
\hline C9 & $0.0234(7)$ & $0.0250(8)$ & $0.0228(7)$ & $0.0008(6)$ & $0.0046(6)$ & $0.0034(6)$ \\
\hline $\mathrm{C} 7$ & $0.0265(7)$ & $0.0237(8)$ & $0.0262(7)$ & $0.0024(6)$ & $0.0088(6)$ & $0.0039(6)$ \\
\hline C31 & $0.0249(7)$ & $0.0172(7)$ & $0.0317(8)$ & $-0.0013(6)$ & $0.0065(6)$ & $0.0014(6)$ \\
\hline C5 & $0.0216(7)$ & $0.0247(7)$ & $0.0219(7)$ & $0.0019(6)$ & $0.0036(6)$ & -0.0015 (6) \\
\hline $\mathrm{C} 11$ & $0.0218(7)$ & $0.0243(7)$ & $0.0240(7)$ & $0.0003(6)$ & $0.0075(6)$ & $-0.0008(6)$ \\
\hline $\mathrm{C} 24$ & $0.0240(7)$ & $0.0232(7)$ & $0.0235(7)$ & $-0.0024(6)$ & $0.0067(6)$ & $-0.0022(6)$ \\
\hline $\mathrm{C} 29$ & $0.0230(7)$ & $0.0202(7)$ & $0.0282(8)$ & $0.0005(6)$ & $0.0094(6)$ & $-0.0007(6)$ \\
\hline $\mathrm{C} 1$ & $0.0269(7)$ & $0.0191(7)$ & $0.0290(8)$ & $-0.0007(6)$ & $0.0040(6)$ & $0.0035(6)$ \\
\hline $\mathrm{C} 26$ & $0.0265(7)$ & $0.0238(7)$ & $0.0230(7)$ & $-0.0016(6)$ & $0.0083(6)$ & $-0.0004(6)$ \\
\hline $\mathrm{C} 30$ & $0.0301(8)$ & $0.0182(7)$ & $0.0328(8)$ & $0.0024(6)$ & $0.0100(6)$ & $-0.0027(6)$ \\
\hline C32 & $0.0222(7)$ & 0.0198 (7) & $0.0269(7)$ & $-0.0009(6)$ & $0.0087(6)$ & $0.0005(6)$ \\
\hline $\mathrm{C} 35$ & $0.0225(7)$ & $0.0235(7)$ & $0.0246(7)$ & $0.0016(6)$ & $0.0075(6)$ & $-0.0008(6)$ \\
\hline $\mathrm{C} 25$ & $0.0269(7)$ & $0.0269(8)$ & $0.0255(7)$ & $-0.0051(6)$ & $0.0074(6)$ & $-0.0006(6)$ \\
\hline C6 & $0.0295(8)$ & $0.0309(8)$ & $0.0279(8)$ & $0.0079(6)$ & $0.0103(6)$ & $0.0009(6)$ \\
\hline $\mathrm{C} 14$ & $0.0253(7)$ & $0.0211(7)$ & $0.0319(8)$ & $0.0009(6)$ & $0.0129(6)$ & $-0.0016(6)$ \\
\hline $\mathrm{C} 15$ & $0.0280(8)$ & $0.0180(7)$ & $0.0360(8)$ & $0.0024(6)$ & $0.0116(6)$ & $-0.0019(6)$ \\
\hline $\mathrm{C} 21$ & $0.0230(7)$ & $0.0297(8)$ & $0.0245(7)$ & $0.0015(6)$ & $0.0053(6)$ & $-0.0068(6)$ \\
\hline C10 & $0.0239(7)$ & $0.0318(8)$ & $0.0261(7)$ & $-0.0002(6)$ & $0.0069(6)$ & $0.0066(6)$ \\
\hline
\end{tabular}


Geometric parameters $\left(\AA,{ }^{\circ}\right)$

\begin{tabular}{|c|c|c|c|}
\hline $\mathrm{O} 5-\mathrm{H} 5 \mathrm{~A}$ & $0.92(2)$ & $\mathrm{C} 18-\mathrm{C} 13$ & 1.4917 (19) \\
\hline O5- $\mathrm{H} 5 \mathrm{~B}$ & $0.81(2)$ & $\mathrm{C} 33-\mathrm{C} 32$ & $1.3899(19)$ \\
\hline $\mathrm{O} 3-\mathrm{H} 3 \mathrm{~A}$ & $0.84(2)$ & $\mathrm{C} 33-\mathrm{C} 34$ & $1.3928(19)$ \\
\hline $\mathrm{O} 3-\mathrm{H} 3 \mathrm{~B}$ & $0.84(2)$ & $\mathrm{C} 28-\mathrm{C} 27$ & $1.3865(19)$ \\
\hline $\mathrm{O} 2-\mathrm{H} 2 \mathrm{~A}$ & $0.86(2)$ & $\mathrm{C} 28-\mathrm{C} 29$ & $1.3926(19)$ \\
\hline $\mathrm{O} 2-\mathrm{H} 2 \mathrm{~B}$ & $0.84(2)$ & $\mathrm{C} 34-\mathrm{C} 35$ & $1.378(2)$ \\
\hline $\mathrm{O} 1-\mathrm{H} 1 \mathrm{~A}$ & $0.77(2)$ & C34-H34 & 0.9300 \\
\hline $\mathrm{O} 1-\mathrm{H} 1 \mathrm{~B}$ & $0.87(2)$ & $\mathrm{C} 8-\mathrm{C} 9$ & $1.392(2)$ \\
\hline $\mathrm{N} 11-\mathrm{C} 38$ & $1.3325(17)$ & $\mathrm{C} 8-\mathrm{C} 7$ & $1.393(2)$ \\
\hline N11-C37 & $1.3332(17)$ & $\mathrm{C} 3-\mathrm{C} 4$ & $1.3904(19)$ \\
\hline $\mathrm{N} 10-\mathrm{C} 36$ & $1.3325(17)$ & $\mathrm{C} 3-\mathrm{C} 2$ & $1.394(2)$ \\
\hline $\mathrm{N} 10-\mathrm{C} 37$ & $1.3395(17)$ & $\mathrm{C} 12-\mathrm{C} 11$ & $1.377(2)$ \\
\hline N6- $\mathrm{C} 18$ & $1.3324(17)$ & $\mathrm{C} 12-\mathrm{C} 13$ & $1.3932(19)$ \\
\hline $\mathrm{N} 6-\mathrm{C} 16$ & $1.3348(17)$ & $\mathrm{C} 12-\mathrm{H} 12$ & 0.9300 \\
\hline $\mathrm{N} 5-\mathrm{C} 18$ & $1.3336(17)$ & $\mathrm{C} 22-\mathrm{C} 21$ & $1.380(2)$ \\
\hline N5-C17 & $1.3350(18)$ & $\mathrm{C} 22-\mathrm{H} 22$ & 0.9300 \\
\hline $\mathrm{O} 4-\mathrm{H} 4 \mathrm{~A}$ & $0.78(2)$ & $\mathrm{C} 4-\mathrm{C} 5$ & $1.378(2)$ \\
\hline $\mathrm{O} 4-\mathrm{H} 4 \mathrm{~B}$ & $0.83(3)$ & $\mathrm{C} 4-\mathrm{H} 4$ & 0.9300 \\
\hline $\mathrm{N} 4-\mathrm{C} 17$ & $1.3338(18)$ & $\mathrm{C} 27-\mathrm{C} 26$ & $1.380(2)$ \\
\hline $\mathrm{N} 4-\mathrm{C} 16$ & $1.3369(17)$ & $\mathrm{C} 27-\mathrm{H} 27$ & 0.9300 \\
\hline $\mathrm{N} 12-\mathrm{C} 36$ & $1.3355(17)$ & $\mathrm{C} 13-\mathrm{C} 14$ & $1.386(2)$ \\
\hline N12-C38 & $1.3378(17)$ & $\mathrm{C} 2-\mathrm{C} 1$ & $1.374(2)$ \\
\hline N9-C31 & $1.3364(19)$ & $\mathrm{C} 2-\mathrm{H} 2$ & 0.9300 \\
\hline N9-C35 & $1.3387(19)$ & $\mathrm{C} 9-\mathrm{C} 10$ & $1.377(2)$ \\
\hline N9- $\mathrm{H} 09$ & 0.8600 & C9-H9 & 0.9300 \\
\hline $\mathrm{N} 3-\mathrm{C} 15$ & $1.3351(19)$ & $\mathrm{C} 7-\mathrm{C} 6$ & $1.377(2)$ \\
\hline $\mathrm{N} 3-\mathrm{C} 11$ & $1.3359(19)$ & $\mathrm{C} 7-\mathrm{H} 7$ & 0.9300 \\
\hline $\mathrm{N} 3-\mathrm{H} 03$ & 0.8600 & $\mathrm{C} 31-\mathrm{C} 32$ & $1.377(2)$ \\
\hline $\mathrm{N} 1-\mathrm{C} 5$ & $1.3336(19)$ & C31-H31 & 0.9300 \\
\hline $\mathrm{N} 1-\mathrm{C} 1$ & $1.3438(19)$ & $\mathrm{C} 5-\mathrm{H} 5$ & 0.9300 \\
\hline $\mathrm{N} 1-\mathrm{H} 01$ & 0.8600 & C11-H11 & 0.9300 \\
\hline $\mathrm{N} 8-\mathrm{C} 26$ & $1.3300(19)$ & $\mathrm{C} 24-\mathrm{C} 25$ & $1.377(2)$ \\
\hline $\mathrm{N} 8-\mathrm{C} 30$ & $1.3439(19)$ & $\mathrm{C} 24-\mathrm{H} 24$ & 0.9300 \\
\hline N8-H08 & 0.8600 & $\mathrm{C} 29-\mathrm{C} 30$ & $1.368(2)$ \\
\hline N7-C21 & $1.334(2)$ & $\mathrm{C} 29-\mathrm{H} 29$ & 0.9300 \\
\hline N7-C25 & $1.3393(19)$ & $\mathrm{C} 1-\mathrm{H} 1$ & 0.9300 \\
\hline N7- $\mathrm{H} 07$ & 0.8600 & $\mathrm{C} 26-\mathrm{H} 26$ & 0.9300 \\
\hline $\mathrm{N} 2-\mathrm{C} 10$ & $1.332(2)$ & $\mathrm{C} 30-\mathrm{H} 30$ & 0.9300 \\
\hline $\mathrm{N} 2-\mathrm{C} 6$ & $1.339(2)$ & C32-H32 & 0.9300 \\
\hline $\mathrm{N} 2-\mathrm{H} 02$ & 0.8600 & C35-H35 & 0.9300 \\
\hline $\mathrm{C} 16-\mathrm{C} 3$ & $1.4848(19)$ & $\mathrm{C} 25-\mathrm{H} 25$ & 0.9300 \\
\hline $\mathrm{C} 36-\mathrm{C} 23$ & $1.4880(19)$ & C6-H6 & 0.9300 \\
\hline $\mathrm{C} 37-\mathrm{C} 28$ & $1.4852(19)$ & $\mathrm{C} 14-\mathrm{C} 15$ & $1.375(2)$ \\
\hline $\mathrm{C} 17-\mathrm{C} 8$ & $1.4883(19)$ & C14-H14 & 0.9300 \\
\hline $\mathrm{C} 38-\mathrm{C} 33$ & $1.4906(18)$ & C15-H15 & 0.9300 \\
\hline $\mathrm{C} 23-\mathrm{C} 22$ & $1.390(2)$ & $\mathrm{C} 21-\mathrm{H} 21$ & 0.9300 \\
\hline
\end{tabular}




\begin{tabular}{|c|c|c|c|}
\hline $\mathrm{C} 23-\mathrm{C} 24$ & $1.394(2)$ & $\mathrm{C} 10-\mathrm{H} 10$ & 0.9300 \\
\hline $\mathrm{H} 5 \mathrm{~A}-\mathrm{O} 5-\mathrm{H} 5 \mathrm{~B}$ & $106.5(19)$ & $\mathrm{C} 21-\mathrm{C} 22-\mathrm{H} 22$ & 120.4 \\
\hline $\mathrm{H} 3 \mathrm{~A}-\mathrm{O} 3-\mathrm{H} 3 \mathrm{~B}$ & $111.1(19)$ & $\mathrm{C} 23-\mathrm{C} 22-\mathrm{H} 22$ & 120.4 \\
\hline $\mathrm{H} 2 \mathrm{~A}-\mathrm{O} 2-\mathrm{H} 2 \mathrm{~B}$ & $106(2)$ & $\mathrm{C} 5-\mathrm{C} 4-\mathrm{C} 3$ & $119.11(13)$ \\
\hline $\mathrm{H} 1 \mathrm{~A}-\mathrm{O} 1-\mathrm{H} 1 \mathrm{~B}$ & $104(2)$ & $\mathrm{C} 5-\mathrm{C} 4-\mathrm{H} 4$ & 120.4 \\
\hline $\mathrm{C} 38-\mathrm{N} 11-\mathrm{C} 37$ & $114.73(11)$ & $\mathrm{C} 3-\mathrm{C} 4-\mathrm{H} 4$ & 120.4 \\
\hline $\mathrm{C} 36-\mathrm{N} 10-\mathrm{C} 37$ & $114.68(11)$ & $\mathrm{C} 26-\mathrm{C} 27-\mathrm{C} 28$ & $119.19(13)$ \\
\hline $\mathrm{C} 18-\mathrm{N} 6-\mathrm{C} 16$ & $114.89(11)$ & $\mathrm{C} 26-\mathrm{C} 27-\mathrm{H} 27$ & 120.4 \\
\hline $\mathrm{C} 18-\mathrm{N} 5-\mathrm{C} 17$ & $114.45(11)$ & $\mathrm{C} 28-\mathrm{C} 27-\mathrm{H} 27$ & 120.4 \\
\hline $\mathrm{H} 4 \mathrm{~A}-\mathrm{O} 4-\mathrm{H} 4 \mathrm{~B}$ & $97(2)$ & $\mathrm{C} 14-\mathrm{C} 13-\mathrm{C} 12$ & $119.43(13)$ \\
\hline $\mathrm{C} 17-\mathrm{N} 4-\mathrm{C} 16$ & $114.62(11)$ & $\mathrm{C} 14-\mathrm{C} 13-\mathrm{C} 18$ & $120.95(12)$ \\
\hline $\mathrm{C} 36-\mathrm{N} 12-\mathrm{C} 38$ & $114.68(11)$ & $\mathrm{C} 12-\mathrm{C} 13-\mathrm{C} 18$ & $119.61(12)$ \\
\hline $\mathrm{C} 31-\mathrm{N} 9-\mathrm{C} 35$ & $122.67(12)$ & $\mathrm{C} 1-\mathrm{C} 2-\mathrm{C} 3$ & 119.19 (13) \\
\hline C31-N9-H09 & 118.7 & $\mathrm{C} 1-\mathrm{C} 2-\mathrm{H} 2$ & 120.4 \\
\hline C35-N9-H09 & 118.7 & $\mathrm{C} 3-\mathrm{C} 2-\mathrm{H} 2$ & 120.4 \\
\hline $\mathrm{C} 15-\mathrm{N} 3-\mathrm{C} 11$ & $122.78(12)$ & $\mathrm{C} 10-\mathrm{C} 9-\mathrm{C} 8$ & 119.09 (14) \\
\hline $\mathrm{C} 15-\mathrm{N} 3-\mathrm{H} 03$ & 118.6 & $\mathrm{C} 10-\mathrm{C} 9-\mathrm{H} 9$ & 120.5 \\
\hline $\mathrm{C} 11-\mathrm{N} 3-\mathrm{H} 03$ & 118.6 & $\mathrm{C} 8-\mathrm{C} 9-\mathrm{H} 9$ & 120.5 \\
\hline $\mathrm{C} 5-\mathrm{N} 1-\mathrm{C} 1$ & $122.81(12)$ & $\mathrm{C} 6-\mathrm{C} 7-\mathrm{C} 8$ & $118.64(14)$ \\
\hline $\mathrm{C} 5-\mathrm{N} 1-\mathrm{H} 01$ & 118.6 & $\mathrm{C} 6-\mathrm{C} 7-\mathrm{H} 7$ & 120.7 \\
\hline $\mathrm{C} 1-\mathrm{N} 1-\mathrm{H} 01$ & 118.6 & $\mathrm{C} 8-\mathrm{C} 7-\mathrm{H} 7$ & 120.7 \\
\hline $\mathrm{C} 26-\mathrm{N} 8-\mathrm{C} 30$ & $122.61(12)$ & $\mathrm{N} 9-\mathrm{C} 31-\mathrm{C} 32$ & $120.12(13)$ \\
\hline $\mathrm{C} 26-\mathrm{N} 8-\mathrm{H} 08$ & 118.7 & $\mathrm{~N} 9-\mathrm{C} 31-\mathrm{H} 31$ & 119.9 \\
\hline $\mathrm{C} 30-\mathrm{N} 8-\mathrm{H} 08$ & 118.7 & $\mathrm{C} 32-\mathrm{C} 31-\mathrm{H} 31$ & 119.9 \\
\hline $\mathrm{C} 21-\mathrm{N} 7-\mathrm{C} 25$ & $122.43(12)$ & $\mathrm{N} 1-\mathrm{C} 5-\mathrm{C} 4$ & $119.79(13)$ \\
\hline $\mathrm{C} 21-\mathrm{N} 7-\mathrm{H} 07$ & 118.8 & $\mathrm{~N} 1-\mathrm{C} 5-\mathrm{H} 5$ & 120.1 \\
\hline $\mathrm{C} 25-\mathrm{N} 7-\mathrm{H} 07$ & 118.8 & $\mathrm{C} 4-\mathrm{C} 5-\mathrm{H} 5$ & 120.1 \\
\hline $\mathrm{C} 10-\mathrm{N} 2-\mathrm{C} 6$ & $122.45(12)$ & $\mathrm{N} 3-\mathrm{C} 11-\mathrm{C} 12$ & $119.64(13)$ \\
\hline $\mathrm{C} 10-\mathrm{N} 2-\mathrm{H} 02$ & 118.8 & $\mathrm{~N} 3-\mathrm{C} 11-\mathrm{H} 11$ & 120.2 \\
\hline $\mathrm{C} 6-\mathrm{N} 2-\mathrm{H} 02$ & 118.8 & $\mathrm{C} 12-\mathrm{C} 11-\mathrm{H} 11$ & 120.2 \\
\hline $\mathrm{N} 6-\mathrm{C} 16-\mathrm{N} 4$ & $125.05(12)$ & $\mathrm{C} 25-\mathrm{C} 24-\mathrm{C} 23$ & $118.58(13)$ \\
\hline $\mathrm{N} 6-\mathrm{C} 16-\mathrm{C} 3$ & $117.42(12)$ & $\mathrm{C} 25-\mathrm{C} 24-\mathrm{H} 24$ & 120.7 \\
\hline $\mathrm{N} 4-\mathrm{C} 16-\mathrm{C} 3$ & $117.52(12)$ & $\mathrm{C} 23-\mathrm{C} 24-\mathrm{H} 24$ & 120.7 \\
\hline $\mathrm{N} 10-\mathrm{C} 36-\mathrm{N} 12$ & $125.28(12)$ & $\mathrm{C} 30-\mathrm{C} 29-\mathrm{C} 28$ & $119.28(13)$ \\
\hline $\mathrm{N} 10-\mathrm{C} 36-\mathrm{C} 23$ & $117.42(11)$ & $\mathrm{C} 30-\mathrm{C} 29-\mathrm{H} 29$ & 120.4 \\
\hline $\mathrm{N} 12-\mathrm{C} 36-\mathrm{C} 23$ & $117.28(11)$ & $\mathrm{C} 28-\mathrm{C} 29-\mathrm{H} 29$ & 120.4 \\
\hline $\mathrm{N} 11-\mathrm{C} 37-\mathrm{N} 10$ & $125.28(12)$ & $\mathrm{N} 1-\mathrm{C} 1-\mathrm{C} 2$ & $119.62(13)$ \\
\hline $\mathrm{N} 11-\mathrm{C} 37-\mathrm{C} 28$ & $117.83(11)$ & $\mathrm{N} 1-\mathrm{C} 1-\mathrm{H} 1$ & 120.2 \\
\hline $\mathrm{N} 10-\mathrm{C} 37-\mathrm{C} 28$ & $116.87(11)$ & $\mathrm{C} 2-\mathrm{C} 1-\mathrm{H} 1$ & 120.2 \\
\hline $\mathrm{N} 4-\mathrm{C} 17-\mathrm{N} 5$ & $125.54(12)$ & $\mathrm{N} 8-\mathrm{C} 26-\mathrm{C} 27$ & $119.79(13)$ \\
\hline $\mathrm{N} 4-\mathrm{C} 17-\mathrm{C} 8$ & $117.00(12)$ & $\mathrm{N} 8-\mathrm{C} 26-\mathrm{H} 26$ & 120.1 \\
\hline $\mathrm{N} 5-\mathrm{C} 17-\mathrm{C} 8$ & $117.46(12)$ & $\mathrm{C} 27-\mathrm{C} 26-\mathrm{H} 26$ & 120.1 \\
\hline $\mathrm{N} 11-\mathrm{C} 38-\mathrm{N} 12$ & $125.32(12)$ & $\mathrm{N} 8-\mathrm{C} 30-\mathrm{C} 29$ & $119.81(13)$ \\
\hline $\mathrm{N} 11-\mathrm{C} 38-\mathrm{C} 33$ & $117.72(11)$ & $\mathrm{N} 8-\mathrm{C} 30-\mathrm{H} 30$ & 120.1 \\
\hline $\mathrm{N} 12-\mathrm{C} 38-\mathrm{C} 33$ & $116.96(11)$ & $\mathrm{C} 29-\mathrm{C} 30-\mathrm{H} 30$ & 120.1 \\
\hline $\mathrm{C} 22-\mathrm{C} 23-\mathrm{C} 24$ & $119.64(13)$ & $\mathrm{C} 31-\mathrm{C} 32-\mathrm{C} 33$ & $118.76(13)$ \\
\hline $\mathrm{C} 22-\mathrm{C} 23-\mathrm{C} 36$ & $120.26(12)$ & $\mathrm{C} 31-\mathrm{C} 32-\mathrm{H} 32$ & 120.6 \\
\hline
\end{tabular}


$120.07(12)$

$125.43(12)$

$117.10(11)$

$117.46(12)$

$119.75(12)$

$120.34(12)$

$119.91(12)$

$119.32(13)$

$120.21(12)$

$120.47(12)$

119.01 (13)

120.5

120.5

$119.53(13)$

$119.69(12)$

$120.77(12)$

$119.46(13)$

$119.71(12)$

$120.82(12)$

119.11 (13)

120.4

120.4

$119.11(13)$

$\begin{array}{ll}\text { C33-C32-H32 } & 120.6 \\ \text { N9-C35-C34 } & 119.68(13) \\ \text { N9-C35-H35 } & 120.2 \\ \text { C34-C35-H35 } & 120.2 \\ \text { N7-C25-C24 } & 120.35(14) \\ \text { N7-C25-H25 } & 119.8 \\ \text { C24-C25-H25 } & 119.8 \\ \text { N2-C6-C7 } & 120.29(14) \\ \text { N2-C6-H6 } & 119.9 \\ \text { C7-C6-H6 } & 119.9 \\ \text { C15-C14-C13 } & 119.17(13) \\ \text { C15-C14-H14 } & 120.4 \\ \text { C13-C14-H14 } & 120.4 \\ \text { N3-C15-C14 } & 119.86(13) \\ \text { N3-C15-H15 } & 120.1 \\ \text { C14-C15-H15 } & 120.1 \\ \text { N7-C21-C22 } & 119.86(13) \\ \text { N7-C21-H21 } & 120.1 \\ \text { C22-C21-H21 } & 120.1 \\ \text { N2-C10-C9 } & 120.00(14) \\ \text { N2-C10-H10 } & 120.0 \\ \text { C9-C10-H10 } & 120.0 \\ & \end{array}$

Hydrogen-bond geometry $\left(\AA,{ }^{\circ}\right)$

\begin{tabular}{|c|c|c|c|c|}
\hline$D-\mathrm{H} \cdots A$ & $D-\mathrm{H}$ & $\mathrm{H} \cdots A$ & $D \cdots A$ & $D-\mathrm{H} \cdots A$ \\
\hline $\mathrm{O} 1-\mathrm{H} 1 A^{\cdots} \cdots \mathrm{Cl} 03^{\mathrm{i}}$ & $0.77(2)$ & $2.47(2)$ & $3.2302(15)$ & $171(2)$ \\
\hline $\mathrm{O} 1-\mathrm{H} 1 B \cdots \mathrm{Cl} 104$ & $0.87(2)$ & $2.32(2)$ & $3.1856(14)$ & $175(2)$ \\
\hline $\mathrm{O} 2-\mathrm{H} 2 A \cdots \mathrm{Cl} 04$ & $0.86(2)$ & $2.28(2)$ & $3.1124(15)$ & $163.2(18)$ \\
\hline $\mathrm{O} 2-\mathrm{H} 2 B \cdots \mathrm{Cl} 05$ & $0.84(2)$ & $2.22(2)$ & $3.0515(13)$ & $170(2)$ \\
\hline $\mathrm{O} 3-\mathrm{H} 3 A \cdots \mathrm{C} 106^{\mathrm{ii}}$ & $0.84(2)$ & $2.22(2)$ & $3.0379(13)$ & $164.5(18)$ \\
\hline $\mathrm{O} 3-\mathrm{H} 3 B \cdots \mathrm{O} 2$ & $0.84(2)$ & $1.91(2)$ & $2.7426(17)$ & $176(2)$ \\
\hline $\mathrm{O} 4-\mathrm{H} 4 A \cdots \mathrm{C} 105^{\mathrm{iii}}$ & $0.78(2)$ & $2.36(2)$ & $3.1356(15)$ & $172(2)$ \\
\hline $\mathrm{O} 4-\mathrm{H} 4 B^{\cdots}{ }^{\cdots} \mathrm{Cl} 03$ & $0.83(3)$ & $2.47(3)$ & $3.2626(14)$ & $162(3)$ \\
\hline $\mathrm{O} 5-\mathrm{H} 5 A \cdots \mathrm{Cl} 06$ & $0.92(2)$ & $2.12(2)$ & $2.9973(12)$ & $157.4(17)$ \\
\hline $\mathrm{O} 5-\mathrm{H} 5 B \cdots \mathrm{Cl} 03$ & $0.81(2)$ & $2.24(2)$ & $3.0466(12)$ & $173(2)$ \\
\hline $\mathrm{N} 1-\mathrm{H} 01 \cdots \mathrm{C} 101^{\mathrm{iv}}$ & 0.86 & 2.24 & $3.0678(12)$ & 161 \\
\hline $\mathrm{N} 2-\mathrm{H} 02 \cdots \mathrm{O} 5$ & 0.86 & 1.77 & $2.5985(16)$ & 162 \\
\hline $\mathrm{N} 3-\mathrm{H} 03 \cdots \mathrm{Cl} 01$ & 0.86 & 2.23 & $3.0405(12)$ & 158 \\
\hline $\mathrm{N} 7-\mathrm{H} 07 \cdots \mathrm{O} 3$ & 0.86 & 1.84 & $2.6472(16)$ & 155 \\
\hline $\mathrm{N} 8-\mathrm{H} 08 \cdots \mathrm{Cl}^{2} 2^{\mathrm{iv}}$ & 0.86 & 2.25 & $3.0732(12)$ & 159 \\
\hline N9-H09 ‥Cl02 & 0.86 & 2.19 & $3.0337(12)$ & 166 \\
\hline $\mathrm{C} 1-\mathrm{H} 1 \cdots \mathrm{Cl} 03^{\mathrm{v}}$ & 0.93 & 2.57 & $3.4995(15)$ & 174 \\
\hline $\mathrm{C} 4-\mathrm{H} 4 \cdots \mathrm{Cl} 04$ & 0.93 & 2.61 & $3.4871(14)$ & 157 \\
\hline $\mathrm{C} 5-\mathrm{H} 5 \cdots \mathrm{O} 1$ & 0.93 & 2.38 & $3.2614(19)$ & 158 \\
\hline $\mathrm{C} 9-\mathrm{H} 9 \cdots \mathrm{Cl}^{2} 6^{\mathrm{v}}$ & 0.93 & 2.70 & $3.4071(15)$ & 134 \\
\hline $\mathrm{C} 10-\mathrm{H} 10 \cdots \mathrm{O} 4$ & 0.93 & 2.54 & $3.370(2)$ & 148 \\
\hline
\end{tabular}


supporting information

$\begin{array}{lllll}\mathrm{C} 11-\mathrm{H} 11 \cdots \mathrm{Cl} 12^{\text {vi }} & 0.93 & 2.61 & 3.5114(15) & 163 \\ \mathrm{C} 12-\mathrm{H} 12 \cdots \mathrm{Cl04} & 0.93 & 2.64 & 3.4992(15) & 154 \\ \mathrm{C} 15-\mathrm{H} 15 \cdots \mathrm{Cl} 03^{\text {vii }} & 0.93 & 2.54 & 3.3157(16) & 141 \\ \mathrm{C} 21-\mathrm{H} 21 \cdots \mathrm{C} 105 & 0.93 & 2.74 & 3.5424(15) & 146 \\ \mathrm{C} 22-\mathrm{H} 22 \cdots \mathrm{Cl}^{\mathrm{v}} 06^{\mathrm{v}} & 0.93 & 2.72 & 3.3883(15) & 130 \\ \mathrm{C} 24-\mathrm{H} 24 \cdots \mathrm{Cl05} 5^{\text {viii }} & 0.93 & 2.63 & 3.4519(15) & 147 \\ \mathrm{C} 26-\mathrm{H} 26 \cdots \mathrm{Cl}^{\text {iix }} & 0.93 & 2.53 & 3.3669(15) & 149 \\ \mathrm{C} 30-\mathrm{H} 30 \cdots \mathrm{O} 2^{\mathrm{x}} & 0.93 & 2.31 & 3.2331(19) & 174 \\ \mathrm{C} 31-\mathrm{H} 31 \cdots \mathrm{O} 2^{\text {viii }} & 0.93 & 2.50 & 3.3320(19) & 149 \\ \mathrm{C} 34-\mathrm{H} 34 \cdots \mathrm{O} 1^{\text {ix }} & 0.93 & 2.50 & 3.3706(19) & 156 \\ \mathrm{C} 35-\mathrm{H} 35 \cdots \mathrm{C} 101^{\text {xi }} & 0.93 & 2.72 & 3.6254(14) & 166\end{array}$

Symmetry codes: (i) $x+1,-y+1 / 2, z-1 / 2$; (ii) $x+1, y$, $z$; (iii) $x-1, y, z$; (iv) $x, y-1, z$; (v) $-x, y-1 / 2,-z+3 / 2$; (vi) $x+1,-y+3 / 2, z-1 / 2$; (vii) $-x, y+1 / 2,-z+3 / 2$; (viii) $-x+1, y+1 / 2,-z+3 / 2$; (ix) $x-1,-y+1 / 2, z+1 / 2$; (x) $-x+1, y-1 / 2,-z+3 / 2$; (xi) $x-1,-y+3 / 2, z+1 / 2$. 